\title{
Interstellar Abundances Toward X Per, Revisited
}

\author{
Lynne A. Valencic \\ Johns Hopkins University, Baltimore, MD 21218; NASA Goddard Space Flight Center, \\ Greenbelt, MD 20771 \\ and \\ Randall K. Smith \\ Smithsonian Astrophysical Observatory, Cambridge, MA 02138
}

Received __ 


\begin{abstract}
The nearby X-ray binary X Per (HD 24534) provides a useful beacon with which to measure elemental abundances in the local ISM. We examine absorption features of $\mathrm{O}, \mathrm{Mg}$, and $\mathrm{Si}$ along this line of sight using spectra from the Chandra Observatory's LETG/ACIS-S and XMM-Newton's RGS instruments. In general, we find that the abundances and their ratios are similar to those of young $\mathrm{F}$ and $\mathrm{G}$ stars and the most recent solar values. We compare our results with abundances required by dust grain models.
\end{abstract}

Subject headings: ISM: abundances 


\section{Introduction}

An integral part of understanding the interstellar medium (ISM) and building realistic dust grain models is determining the abundances of the elements in the dust phase. The most common method is to measure this indirectly, that is, to measure the gas-phase abundance and subtract this from the total (gas+dust) abundance. The gas-phase component of the local ISM is fairly well-known, having been determined by UV/optical absorption studies by many workers (Cardelli et al. 1996; Sofia et al. 1997; Cardelli \& Meyer 1997; Sofia et al. 1998; Jensen et al. 2005; Gnacinski \& Krogulec 2006) and appears to be relatively independent of the line of sight used. However, the total local ISM elemental abundances are not well known, and proxies must be used instead.

Historically, the Sun has been used as the standard for ISM abundances. However, while most solar abundances have remained more or less constant over the years, those of the main dust-forming elements have been revised downward considerably (Anders \& Grevesse 1989; Grevesse \& Sauval 1998; Asplund et al. 2005; Asplund et al. 2009). The recent revisions have brought solar O into better agreement with inferred ISM abundances of Wilms et al. (2000) and Sofia \& Meyer's (2001) young F and G stars. We note, though, that large scatter remains and there is no particularly persuasive reason why the $4.5 \mathrm{Gyr}$ Sun should be an accurate representation of the ISM today.

B stars and metal-rich F and G stars have also been suggested as proxies for the ISM but these have problems of their own. Hempel \& Holweger (2003) showed that B stars cannot be considered an accurate indicator of ISM abundances, as element stratification due to diffusion appears common in these stars. Thus the abundances measured at their surfaces do not necessarily reflect the abundances of the clouds from which they formed, though the latest revision of B star abundances seem to ease differences between them and solar values somewhat (Nieva \& Simón-Díaz 2011). Regarding F and G stars, Edvardsson 
et al.'s (1993) study of stars in the solar neighborhood showed that there is large scatter in the their age-metallicity relation; thus, metal-rich F and G stars are not necessarily young, and cannot be used as a gauge of today's ISM abundances. Sofia \& Meyer (2001), using Edvardisson et al.'s (1993) sample, calculated the average abundances for F and G stars less than 2 Gyr old. They found values for $\mathrm{Mg}$ and $\mathrm{Si}$ that are similar to the most recent solar values, but less $O$ than solar (Asplund et al. 2009) Some have suggested using abundances measured in $\mathrm{H}$ II regions, but this has been rejected due to large systematic errors from temperature fluctuations in the nebula (Mathis 1995; Kingdon \& Ferland 1995). Further complicating the situation, and highlighting the need for spectroscopy on nearby objects, is the correlation between Galactocentric distance and abundances (Shaver et al. 1983; Gummersbach et al. 1998; Chiappini et al. 2001), as regions closer to the Galactic center are more metal-rich than outer regions.

A direct measurement of the local ISM is the best way to determine exactly how much material remains for local dust grains. X-ray spectroscopy is particularly useful for this, as the elements which make up the grains have their K- and L- shell absorption edges in this band. The edge depths are dependent on each element's total column density (gas + dust phases), while fine structure near the edges can determine the chemical state. Schattenburg \& Canizares (1986) were the first to measure X-ray absorption edges; they used the Einstein Observatory to study the $\mathrm{K}$ edges of $\mathrm{O}$ and $\mathrm{Ne}$ toward the Crab Nebula. Since then, much $\mathrm{X}$-ray spectroscopy has been done, usually toward very bright (and distant) low mass X-ray binaries. Paerels et al. (2001) used the Chandra X-ray Observatory's LETG to measure the column densities of $\mathrm{O}, \mathrm{Ne}$, and Fe toward 4U0614+091, at a distance of about 1.5 kpc (Machin et al. 1990). Takei et al (2002) examined the O edge and its fine structure along the line of sight toward Cyg X-2, and Ueda et al. (2005) determined the abundances of $\mathrm{O}, \mathrm{Mg}$, Si, S, and Fe toward GX 13+1, GX 5-1, and GX 340+0. Juett et al. (2004, 2006) examined the $\mathrm{K}$ edge and fine structure of $\mathrm{O}, \mathrm{Ne}$, and $\mathrm{Fe}$ for several x-ray binaries. 
Constantini et al. (2010) used XMM-Newton, INTEGRAL, and Chandra to study the ISM toward 4U1820-30, focusing on fitting fine structure near the Fe L and $\mathrm{O} K$ edges with dust constituent spectra derived from experiment. However, these studies focus on long lines of sight. For instance, 4U1820-30 is located in the globular cluster NGC 6624, between 6.8-9.6 kpc away (G̈uver et al. 2010). Cyg X-2 is $1.6 \mathrm{kpc}$ away (Goranskij \& Lyutyj 1988), and GX 13+1, GX 5-1, and GX 340+0 are near the Galactic center, 7, 9.2, and $11.0 \mathrm{kpc}$ away, respectively (Jonker et al. 2000). 4U0614+091 is about $3.2 \mathrm{kpc}$ away (Kuulkers et al. 2010).

In contrast, Cunningham et al. (2004) used the Chandra LETG to examine the line of sight toward X Per, only $\sim 400 \mathrm{pc}$ away. While they were able to measure the $\mathrm{O}$ column density, the observation unfortunately had low signal-to-noise, leading to an uncertainty of about $50 \%$ in their measurement and an inability to impose meaningful constraints on grain models. Thus, to better assess the elelmental budget in the nearby ISM, in particular that of silicate-forming dust, we have obtained and analyzed high-resolution X-ray spectra of X Per from both Chandra and XMM-Newton.

\section{The X Per System}

$\mathrm{X}$ Persei (HD 24534, BD+30 591) is a Be/X-ray binary is composed of a neutron star and OB companion of likely spectral type O9.5III to B0V (Liu et al. 2006; Raguzova 2007). It is variable, with $m_{V}=6.1-6.8$. The optical variations are believed to be due to the formation and dissipation of an equatorial disk (Telting et al. 1998), similar to other such Be binary systems. The neutron star has a rotation period of about $835 \mathrm{~s}$, one of the longest observed for a neutron star (Haberl et al. 1998). Distance estimates from spectroscopic parallax have placed it as far away as $1.3 \mathrm{kpc}$ (e.g. Fabregat et al. 1992) but more recent work indicates it is closer, at $\sim 0.7-1 \mathrm{kpc}$ (Lyubimkov et al. 1997; Roche et al. 1997;

Telting et al. 1998). Hipparcos parallax places it at $427_{-98}^{+183} \mathrm{pc}$, in agreement with Blaauw's 
(1952) assessment that it is in the $\zeta$ Per OB association ( $d=350$ pc; Johnson 1959).

The ISM toward X Per has also been studied extensively, and it happens that this sight line is an excellent representative for the diffuse ISM at large, as seen through the ratio of total-to-selective extinction $\mathrm{R}_{V}\left(\equiv \mathrm{A}_{V} /(\mathrm{E}(\mathrm{B}-\mathrm{V})) . \mathrm{R}_{V}\right.$ is a general indicator of grain size and local ambient radiation field; it is the sole variable upon which differences in UV extinction curves depend (Cardelli et al. 1989). It ranges from $\sim 2.5$ (diffuse sight line) to $\sim 5.5$ (dense sight line), with the "standard diffuse ISM" having $\mathrm{R}_{V}=3.05 \pm 0.15$ (Whittet 2003); most grain models assume this standard value. It is identical to $\mathrm{R}_{V}$ for the $\mathrm{X}$ Per line of sight, 3.06 (Valencic \& Smith 2008), making X Per ideal for studying the local ISM.

\section{Observation and Data Reduction}

We used two observations, one from Chandra's LETG/ACIS-S and the other from XMM-Newton's RGS. The Chandra observation (Obs ID 12447) had an exposure time of 131 ks. We reprocessed the data with CIAO 4.3 (CALDB 4.4.1) according to standard methods for grating spectra as described in the Science Threads ${ }^{1}$ using the task "chandra_repro" to generate a new bad pixel file, event file, and PHA files. Before continuing, we examined the light curve and found that it did not show any flaring during this time, and only slight variability, with an average count rate of $0.96 \pm 0.10$ count/sec. We then created and applied the response and ancillary files for the +1 and -1 orders, which we coadded and grouped to ensure a minimum of 15 counts per bin (Cunningham et al. 2005), which led to a binsize of $0.007 \mathrm{keV}$ over the spectrum. The XMM-Newton observation (Obs ID 0600980101) had an exposure time of $126 \mathrm{ks}$. We reprocessed the RGS data with SAS 11,

\footnotetext{
${ }^{1}$ http://cxc.cfa.harvard.edu/ciao/
} 
according to standard procedures described in the "ABC Guide"2. After reprocessing, we examined the light curve, which showed flaring at the start and end of the observation; once we excised those events, the effective exposure time was $\sim 100 \mathrm{ks}$. We made and applied response files for the first order spectra and rebinned to ensure a minimum of 15 counts per bin, which led to a binsize of $0.0013 \mathrm{keV}$ over the spectrum. We did not make ancillary files, as these are not needed for RGS data. We also did not combine the spectra from the two RGS instruments. In the analysis, we examined the LETG data first, as it typically has a higher effective area than RGS.

Unfortunately, the signal-to-noise was too low to reliably detect fine structure. The edges were measured as described below. We used the cross sections of Verner \& Yakovlev (1995) and the total $\mathrm{H}$ column density from Cunningham et al. (2004), $\mathrm{N}_{\mathrm{H}}=$ $2.19 \times 10^{21} \mathrm{~cm}^{-2}$, to calculate the number of atoms per million $\mathrm{H}$ nuclei. The uncertainties given with our results are the $1 \sigma$ statistical errors. Table 1 lists our results and the amount of material in the gas and dust phases, where the gas phase abundances are from the literature. Fig. 4 compares our results to those of other workers.

\subsection{Oxygen}

We began by examining the LETG data following the method described by Juett et al. (2004), fitting the continuum around the edge with an absorbed power law, and freezing the continuum while fitting the edge explicitly. This was done over a range of energies (Takei et al. 2002). We used the tbvarabs model (Wilms et al. 2000 ), holding $\mathrm{N}_{\mathrm{H}}=2.19 \times 10^{21} \mathrm{~cm}^{-2}$ and $\mathrm{O}$ abundance at 0 , and measuring the actual $\mathrm{O}$ absorption with an additional edge model. While the spectrum had suggestions of fine structure, in particular for the atomic $\mathrm{O}$

\footnotetext{
${ }^{2}$ http://heasarc.nasa.gov/docs/xmm/abc/
} 
absorption line at $0.527 \mathrm{keV}$, the signal-to-noise was too low to fit the feature. Therefore, we restricted ourselves to a simple edge fit and did not include the $0.525-0.548 \mathrm{keV}$ region in the continuum fit (Takei et al. 2002; Cunningham et al. 2005). This also minimized confusion with instrumental effects. We tried to avoid absorption features from other elements as much as possible, specifically from the Fe L II and III edges at 0.71 and 0.72 $\mathrm{keV}$, respectively, and $\mathrm{Ne}$ at $0.87 \mathrm{keV}$. While no absorption from Fe L edges was visible, we nonetheless tested the dependence of the $\mathrm{O}$ optical depth on the Fe abundance by assuming Fe abundances of $0.5,1.0$, and $2.0 \times$ the values of Wilms et al. (2000) (Takei et al. 2002) and following the same fitting procedure described above. This led to a maximum difference in optical depth of about $4 \%$, or $30 \%$ of the statistical error. We therefore kept the Fe abundance fixed at the value from Wilms et al. (2000). We found that fits with a minimum energy of $0.44-0.46 \mathrm{keV}$ and a maximum energy of $0.69-0.73 \mathrm{keV}$ consistently produced edge energies and optical depths that were within $1 \sigma$ of each other, with edge energies of $0.537 \pm 0.010 \mathrm{keV}$ to $0.541 \pm 0.010 \mathrm{keV}$, and optical depths between $0.54 \pm 0.03$ and $0.57 \pm 0.03$. Values of the reduced $\chi_{\nu}^{2}$ ranged from 0.68 to 0.72 , with the best values corresponding to optical depths of $0.54-0.56$ and an edge energy of $0.538 \pm 0.010 \mathrm{keV}$.

We also examined the edge in the RGS spectrum, following the same procedure. We used only RGS1, as CCD4 in RGS2, which covers $0.514-0.620 \mathrm{keV}$, was lost early in the mission. Over the same energy ranges, we found the edge energies of $0.538 \pm 0.010 \mathrm{keV}$ to $0.540 \pm 0.010 \mathrm{keV}$. The optical depths and $\chi_{\nu}^{2}$ values were in the same ranges as for LETG, and the best fits corresponded to optical depths of 0.54-0.55 \pm 0.03 . Using an optical depth of $0.54 \pm 0.02$, we find $\mathrm{N}_{\mathrm{O}}=(1.09 \pm 0.04) \times 10^{18} \mathrm{~cm}^{-2}$, and $\mathrm{N}_{\mathrm{O}} / \mathrm{N}_{\mathrm{H}}=(4.95 \pm 0.18) \times 10^{-4}$. Our value for the edge energy is lower than expected for $\mathrm{O}_{2}$ and atomic oxygen $(0.543$ and $0.544 \mathrm{keV}$, respectively; Sevier 1979; Stolte et al. 1997) and in agreement with that of metal oxides (0.532 0.540 keV; Sevier 1979; Nakai et al. 1987) and molecules containing O-C single bonds (0.538-0.542 keV; Yang et al. 1987). Our value for $\mathrm{N}_{\mathrm{O}}$ is slightly higher than 
those found by Cunningham et al. (2004), though we still agree within $3 \sigma$; they found $\sim$. $7-8_{-4.0}^{+3.7} \times 10^{17} \mathrm{~cm}^{-2}$. The fits from $0.45-0.70 \mathrm{keV}$ are shown in Fig. 1.

\subsection{Magnesium}

We began by examining the LETG data, which does not have internal Mg features, unlike the RGS. Laboratory spectra of $\mathrm{MgO}$ show structure between $1.31-1.33 \mathrm{keV}$ (i.e. Luches et al. 2004, Limpijumnong et al. 2011), with the K edge near $1.31 \mathrm{keV}$. No such structure was visible; but we nonetheless excluded this region from our fits to minimize any effect it might have. We followed the same method as for $\mathrm{O}$ : we fitted the continuum around the edge, exluding the region from $1.31-1.33 \mathrm{keV}$, over a range of minimum and maximum energies. We again let the continuum be an absorbed power law, with $\mathrm{N}_{\mathrm{H}}=$ $2.19 \times 10^{21} \mathrm{~cm}^{-2}$ in tbvarabs and the $\mathrm{Mg}$ abundance set to 0 . For each energy range, we froze the continuum and tried to fit the edge, but the fits consistently could not find it. We then inciuded the 1.31-1.33 keV range and allowed the continuum parameters to float; again, the fits could not consistently find the edge. We then froze the edge energy at $1.307 \mathrm{keV}$ (Ueda et al. 2005) and re-fitted the spectrum. We found that for minimum energies from 1.25-1.27 keV and maximum energies from 1.36-1.38 keV, the optical depth ranged from $0.016-0.024 \pm 0.008$, with the best fits $\left(\chi_{\nu}^{2}=0.94\right)$ consistently producing an optical depth of $0.020 \pm 0.007$.

We then analyzed the RGS data. The RGS2 has a chip edge at $1.24 \mathrm{keV}$, near the $\mathrm{Mg}$ $\mathrm{K}$ edge, so we restricted ourselves to the RGS1 to minimize any effect this might have on

the fit. We again let the continuum be an absorbed power law, with $\mathrm{N}_{\mathrm{H}}=2.19 \times 10^{21} \mathrm{~cm}^{-2}$ in tbvarabs and set the $\mathrm{Mg}$ abundance to 0 . We again began by fitting the continuum around the edge, exluding the region from 1.31-1.33 keV, over a range of minimum and maximum energies. For each energy range, we then froze the continuum and tried to fit the 
edge, but this consistently yielded opticai depths smaller than the uncertainies. Despite the possibility of introducing instrumental effects, we then included the $1.31-1.33 \mathrm{keV}$ region in the fits and allowed the continuum parameters to float. For the same range of minimum and maximum energies, we consistently found an edge energy that was within $1 \sigma$ of the LETG result, $1.309 \pm 0.007$, and the best fits $\left(\chi_{\nu}^{2}=1.19\right)$ corresponded to an optical depth of $0.019 \pm 0.013$. For an optical depth of $0.020 \pm 0.007$, we find $\mathrm{N}_{\mathrm{Mg}}=(9.86 \pm 3.45) \times 10^{16} \mathrm{~cm}^{-2}$ and $\mathrm{N}_{\mathrm{Mg}} / \mathrm{N}_{\mathrm{H}}=(4.50 \pm 1.58) \times 10^{-5}$. The fits from $1.25-1.38 \mathrm{keV}$ are shown in Fig. 2.

\subsection{Silicon}

We again fitted the LETG spectrum by treating the continuum as an absorbed power law with $\mathrm{N}_{\mathrm{H}}$ at $2.19 \times 10^{21} \mathrm{~cm}^{-2}$ in tbvarabs and setting the $\mathrm{Si}$ abundance to 0 in tbvarabs. We excluded the region $1.84-1.87 \mathrm{keV}$ in an effort to minimize any confusion with instrumental effects (Ueda et al. 2005), and allowed all parameters to float. We found that fits with a minimum energy $1.75-1.78 \mathrm{keV}$ and maximum energy $1.93-1.95 \mathrm{keV}$ produced the best fits, with $\chi_{\nu}^{2}$ between $0.97-1.03$ and values for the optical depth of $0.007 \pm 0.006$ to $0.010 \pm 0.006$ and edge energy of $1.846 \pm 0.015$ to $1.851 \pm 0.015$. The best values of $\chi_{\nu}^{2}$ corresponded to an optical depth of $0.010 \pm 0.006$. The fit from $1.75-1.95 \mathrm{keV}$ is shown in Fig. 3.

We again tried to verify this measurement with the RGS data using the same energy ranges and method. We fitted the R1 and R2 spectra simultaneously, and found no excess absorption at the Si edge. However, the R1 and R2 have bad pixels near the Si edge, which could lead to underestimates of the continuum. To see if these were having an effect on the measurement, we then fitted the R1 and R2 spectra seperately. Again, no excess absorption was detected.

We then attempted to verify the origin of the additional absorption (ISM or instrument) 
in the Chandra spectrum by examining an unabsorbed line of sight. We examined a Chandra LETG/ACIS-S spectrum of Mkn 421 (Obs ID 5171), a line of sight known to have very little interstellar material. We used the HEASARC's Galactic HI column density calculator to estimate $\mathrm{N}_{\mathrm{H}}$ within a $1^{\circ}$ radius of Mkn 421 . The Galactic HI survey of Dickey \& Lockman (1990) indicates $\mathrm{N}_{\mathrm{H}} \sim 1.4 \times 10^{20} \mathrm{~cm}^{-2}$, and the Leiden/Argentine/Bonn Survey (Kalberla et al. 2005) shows $\mathrm{N}_{\mathrm{H}} \sim 1.9 \times 10^{20} \mathrm{~cm}^{-2}$, both of which are lower than that of $\mathrm{X}$ Per's line of sight by an order of magnitude. We reprocessed the Mkn 421 data, extracted the spectrum, and examined it about the Si edge using the same methods and over the same energy ranges as the X Per spectrum. No excess absorption was found, so despite the smallness of the measurement for the $\mathrm{X}$ Per line of sight, we believe it represents the contribution from the ISM. Thus, for an optical depth of $0.010 \pm 0.006$, we find $\mathrm{N}_{\mathrm{Si}}=$ $(7.37 \pm 4.42) \times 10^{16} \mathrm{~cm}^{-2}$ and $\mathrm{N}_{\mathrm{Si}} / \mathrm{N}_{\mathrm{H}}=(3.37 \pm 2.02) \times 10^{-5}$.

\section{Discussion}

In general, our results tend to be similar to those found by other studies of the local ISM and the most recent proto-solar nebula estimates, as determined by the diffusion-corrected photospheric values of Asplund et al. (2009), which has brought solar O values closer to those of young F and G stars (Sofia \& Meyer 2001). Fig. 4 shows our results and those of other workers for comparison. Abundances of $\mathrm{O}$ show a fair amount of scatter, from 251 atoms $/ 10^{6} \mathrm{H}$ in meteorites (Lodders et al. 2009) to 851 atoms $/ 10^{6} \mathrm{H}$ in the solar value from Anders \& Grevesse (1989). However, recent studies show $O$ abundances between these values. A re-analysis of 13 B stars in Ori OB1 by Nieva \& Simón-Díaz (2011) indicated there are $589 \mathrm{O}$ atoms $/ 10^{6} \mathrm{H}$, though another study by Simón-Díaz (2010) places the O abundance for the same 13 stars at 537 atoms $/ 10^{6} \mathrm{H}$. Our value of 495 atoms $/ 10^{6} \mathrm{H}$ is nearly identical to that of Wilms et al. (2000) and agrees within $3 \sigma$ of Sofia \& Meyer's F 
and G stars, Simón-Díaz's (2010) B stars, and Asplund et al.'s (2009) solar nebula estimate.

There is not nearly so much scatter in Mg estimates, which tend to range from about 25 to 40 atoms $/ 10^{6} \mathrm{H}$. While our result for $\mathrm{Mg}$ is high (45.0 15.8 ), it is nonetheless similar to that of Asplund et al. (2009) and Sofia \& Meyer (2001) (43.7 $7_{-3.9}^{+4.2}$ and $42.7 \pm 17.2$, respectively). Estimates for $\mathrm{Si}$ also tend to cover a narrow range, about 20 to 40 atoms $/ 10^{6}$ $\mathrm{H}$. As with $\mathrm{Mg}$, our result is similar to that found by Asplund et al. (2009) and Sofia \& Meyer (2001), as well as Simón-Díaz (2010).

Seeing the agreement between our abundance determinations and those of other workers, in particular that of Asplund et al. (2009), Sofia \& Meyer (2001), and Simón-Díaz (2010), it is not surprising that our abundance ratios are similar to theirs, too. Fig. 5 compares our ratios to those of the same workers from Fig. 4.

We also compared our results to those made toward other X-ray binaries. Juett et al. (2004), measured the $\mathrm{O}$ column density and examined structure near the $\mathrm{O}$ edge in seven lines of sight toward bright X-ray binaries. In order to estimate the total $\mathrm{N}_{\mathrm{H}}$, we made use of Takei et al.'s (2002) finding that for the diffuse ISM $\left(\mathrm{N}_{\mathrm{HI}}=2.17 \times 10^{21} \mathrm{~cm}^{-2}\right)$ toward Cyg X-2, $\mathrm{N}_{\mathrm{H}_{2}} \lesssim 5 \% \mathrm{~N}_{\mathrm{HI}}$ and $\mathrm{N}_{\mathrm{HII}} \sim 32 \% \mathrm{~N}_{\mathrm{HI}}$. We again used the Galactic HI column density calculator and the Leiden/Argentine/Bonn Survey. (Kalberla et al. 2005) to find $\mathrm{N}_{\mathrm{HI}}$ within $1^{\circ}$ for each of the sources, then found the total $\mathrm{H}$ column density. This resulted in $\mathrm{O}$ abundances from $378-916$ atoms $/ 10^{6} \mathrm{H}$. Unsurprisingly, our abundances are notably lower than those along the sight lines toward the Galactic Center (Ueda et al. 2005). Using Ueda et al.'s (2005) total $\mathrm{N}_{\mathrm{H}}$ values for GX 13+1, GX 5-1, and GX 340+0, $\left(2.1_{-1.3}^{+3.4} \times 10^{22}, 2.8_{-1.8}^{+3.3} \times 10^{22}\right.$, and $4.8_{-2.9}^{+5.0} \times 10^{22} \mathrm{~cm}^{-2}$, respectively $)$, we found that $\mathrm{O}$ ranged from 833-857 atoms $/ 10^{6} \mathrm{H}, \mathrm{Mg}$ ranged from $75-86$ atoms $/ 10^{6} \mathrm{H}$, and $\mathrm{Si}$ ranged from $60-75$ atoms $/ 10^{6} \mathrm{H}$. In the case of GX 5-1, Smith et al. (2006) found that $\mathrm{N}_{\mathrm{H}}$ could be as high as $3.3 \times 10^{22} \mathrm{~cm}^{-2}$, depending on the brightness of a CO cloud along the sight line. This 
higher $\mathrm{N}_{\mathrm{H}}$, while lowering the abundances commensurately, did not lower them sufficiently to bring them into agreement with values from the local ISM: 727, 72, and 55 atoms/10 $\mathrm{H}$ for $\mathrm{O}, \mathrm{Mg}$, and $\mathrm{Si}$, respectively. Our $\mathrm{O}$ abundance is also substantially lower than that which Costantini et al. (2012) found for the line of sight toward 4U1830-20, 755 atoms $/ 10^{6}$ $\mathrm{H}$.

Using our total abundances, and those from the gas phase from the literature, we can find how much is depleted into dust. These are shown in Table 1 . Only about $38 \%$ of the total $\mathrm{O}$ for this line of sight is in the dust phase, while $\mathrm{Mg}$ and $\mathrm{Si}$ are almost completely depleted (97\%). This is in accordance with what has been found for other diffuse lines of sight (Savage \& Sembach 1996; Whittet 2003). Snow \& Witt (1996) examined the abundance requirements of nine grain models; these are listed in their Table 3. In a similar manner, we examine more recent models in our Table 2, and include their findings. We also include the dust phase abundances found in this work for ease of comparison. For models where only $\mathrm{Si}$ is listed, we assumed that the $\mathrm{O}$ requirements were four times that of $\mathrm{Si}\left(\mathrm{SiO}_{4}\right)$ (Snow \& Witt 1996; Cunningham et al. 2004) and for models where $\mathrm{Mg}$ was not listed and the type of silicate was not specified, we assumed that the model needed an amount comparable to $\mathrm{Si}$ (Snow \& Witt 1996). Abundance requirements found in this way are in parentheses.

For this line of sight, there appears to be sufficient $O$ for most models to within 1 $\sigma$. Unfortunately, the large uncertainties in our $\mathrm{Mg}$ and $\mathrm{Si}$ measurements do not allow us to meaningfully constrain these quantities. We can only note that there appears to be sufficient $\mathrm{Mg}$ for most models, and less $\mathrm{Si}$ appears available than needed by the models of Weingartner \& Draine (2001) and Iati et al. (2008). 


\section{Conclusions}

We have obtained and analyzed spectra of the short line of sight toward X Per using the Chandra and XMM-Newton X-ray observatories. X-ray spectroscopy shows the abundances of silicate dust-forming elements are largely similar to the latest estimates from classical proxies. The $\mathrm{O}$ abundance is substantially higher than that determined from meteorites. While the uncertainties in the $\mathrm{Mg}$ and $\mathrm{Si}$ measurements are large, our findings suggest that the abundances of these elements are more similar to solar, young F and G star, and the latest B star estimates than previously inferred ISM values. Higher signal-to-noise observations are needed to reduce the large uncertainties in the $\mathrm{Mg}$ and $\mathrm{Si}$ measurements and place meaningful constraints on dust grain models.

Facilities: CXO (ACIS), XMM-Newton (RGS) 


\section{REFERENCES}

Anders, E. \& Grevesse, N. 1989, Geochim. Cosmochim Acta, 53, 197

Asplund, M., Grevesse, N., \& Sauval, M. 2005, in ASP Conf. Ser. 336, Cosmic Abundances as Records of Stellar Evolution and Nucleosynthesis in honor of David L. Lambert, ed. T. G. Barnes III \& F. N. Bash (San Francisco, CA: ASP), 25

Asplund M., Grevesse, N., Sauval, A. J., \& Scott, P. 2009, ARA\&A, 47, 481

Blaauw, A. 1952, Bull. Astron. Inst. Netherlands, 11, 405

Cardelli, J. A., Meyer, D. M., Jura, M., \& Savage, B. D. 1996, ApJ, 467, 334

Cardelli, J. A., \& Meyer, D. M. 1997, ApJL, 477, 57

Cardelli, J. A., Clayton, G. C., \& Mathis, J. S. 1989, ApJ, 345, 245

Cecchi-Pestellini, C., Cacciola, A., Iatì, M. A., et al. 2010, MNRAS, 408, 535

Chiappini C., Matteucci, F., \& Romano, D. 2001, ApJ, 554, 1044

Costantini, E., Pinto, C., Kaastra, J. S., et al. 2012, A\&A, 539, 32

Cunningham, N. J., McCray, R., \& Snow, T. P. 2004, ApJ, 611, 353

Dickey, J. M. \& Lockman, F. J. 1990, ARA\&A, 28, 215

Edvardsson, B., Andersen, J., Gustafsson, B., et al. 1993, A\&AS, 275, 101

Fabregat, J., Reglero, V., Coe, M. J., et al. 1992, A\&A, 259, 522

Gnacinski, P. \& Krogulec M. 2006, Acta Astr. 56, 373

Goranskij, V. P. \& Lyutyj, V. M. 1988, SvA, 32, 193 
Grevesse, N. \& Sauval, A. J. 1998, SSRev, 85, 161

Gummersbach, C. A., Kaufer, A., Schaefer, D. R., et al. 1998, A\&A, 338, 881

Güver, T., Wroblewski, P., Camarota, L., \& Özel, F. 2010, ApJ, 719, 1807

Haberl, F., Angelini, L., Motch, C., \& White, N. E. 1998, A\&A, 330, 189

Hempel, M. \& Holweger, H. 2003, A\&A, 408, 1065

Iatì, M. A., Saija, R., Borghese, F., et al. 2008, MNRAS, 384, 591

Jensen, A. G., Rachford, B. L., \& Snow, T. P. 2005, ApJ, 619, 891

Johnson, H. L. 1957, ApJ, 126, 121

Juett, A. M., Schulz, N. S., \& Chakrabarty, D. 2004, ApJ, 612, 308

Juett, A. M., Schulz, N. S., Chakrabarty, D., \& Gorczyca, T. W. 2006, ApJ, 648, 1066

Kalberla, P. M. W., Burton, W. B., Hartmann, D., et al. 2005, A\&A, 440, 775

Kingdon, J. B. \& Ferland, G. J. 1995, ApJ, 450, 691

Kuulkers, E., in't Zand, J. J. M., Atteia, J.-L., et al. 2010, A\&A, 514, 65

Limpijumnong, S., Jutimoosik, J., Palakawong, N., et al. 2011, Appl. Phys. Lett., 99, 261901

Liu, Q. Z., van Paradijs J., \& van den Heuvel E.P.J. 2006, A\&A, 455, 1165

Lodders, K., Palme, H, \& Gail, H.-P. 2009, in Landolt-Börnstein, New Series, vol VI/4B, ed. J. E. Trümper (Berlin: Springer), 560

Luches, P. D'Addato, S., Valeri, S., et al. 2004, Phys. Rev. B, 69, 045412 
Lyubimkov, L. S., Rostopchin, S. I., Roche, P., Tarasov, A. E. 1997, MNRAS, 286, 549

Møchin, G., Callanan, P. J., Charles, P. A., et al. 1990, MNRAS, 247, 205

Mathis, J. S. 1995, Rev. Mex. AA Ser. Conf. 3, 207

Nakai, S.-I., Mitsuishi, T., Sugawara, H., et al. 1987, Phys. Rev. B, 36, 9241

Nieva, M.-F. \& Simón-Díaz, S. 2011, A\&A, 532, 2

Paerels, F., Brinkman, A. C., van der Meer, R. L. J., et al. 2001, ApJ, 546, 338

Raguzova, N.V. 2007, BeSN, 38, 24

Roche, P., Larionov, V., Tarasov, A. E., et al. 1997, A\&A, 322, 139

Savage, B. D. \& Sembach, K. R. 1996, ARA\&A, 34, 279

Schattenburg, M. L. \& Canizares, C. R. 1986, ApJ, 301, 759

Sevier, K. D. 1979, At. Data Nucl. Data Tables, 24, 323

Simón-Díaz, S. 2010, A\&A, 510, 22

Shaver, P. A.; McGee, R. X.; Newton, L. M.; Danks, A. C.; Pottasch, S. R. 1983, MNRAS, 204,53

Smith, R. K., Dame, T. M., Costantini, E., \& Predehl, P. 2006, ApJ, 648, 452

Snow, T. P. \& Witt, A. N. 1996, ApJL, 468, 65

Sofia, U. J. \& Meyer, D. M. 2001, ApJL, 554, 221

Stolte, W. C., Lu, Y., Samson, J. A. R. et al. 1997, J. Phys. B, 30, 4489

Takei, Y., Fujimoto, R., Mitsuda, K., \& Onaka, T. 2002, ApJ, 581, 307 
Telting, J. H., Waters, L. B. F. M., Roche, P., et al. 1998, MNRAS, 296, 785

Ueda, Y., Mitsuda, K., Murakami, H., \& Matsushita, K. 2005, ApJ, 620, 274

Vaidya, D. B., Gupta, R., \& Snow, T. P. 2007, MNRAS, 379, 791

Valencic, L. A. \& Smith, R. K. 2008, ApJ, 672, 984

Verner, D. A. \& Yakovlev, D. G. 1995, A\&AS, 109, 125

Weingartner, J. C. \& Draine, B. T. 2001, ApJ, 548, 296

Whittet, D. C. B. 2003, Dust in the Galactic Environment (2nd Ed.; Philadelphia: IOP)

Wilms, J., Allen, A., \& McCray, R. 2000, ApJ, 542, 914

Yang, B. X., Kirz, J., \& Sham, T. K. 1987, Phys. Rev. A, 36, 4298

Zubko, V., Dwek, E., \& Arendt, R. G. 2004, ApJS, 152, 211 

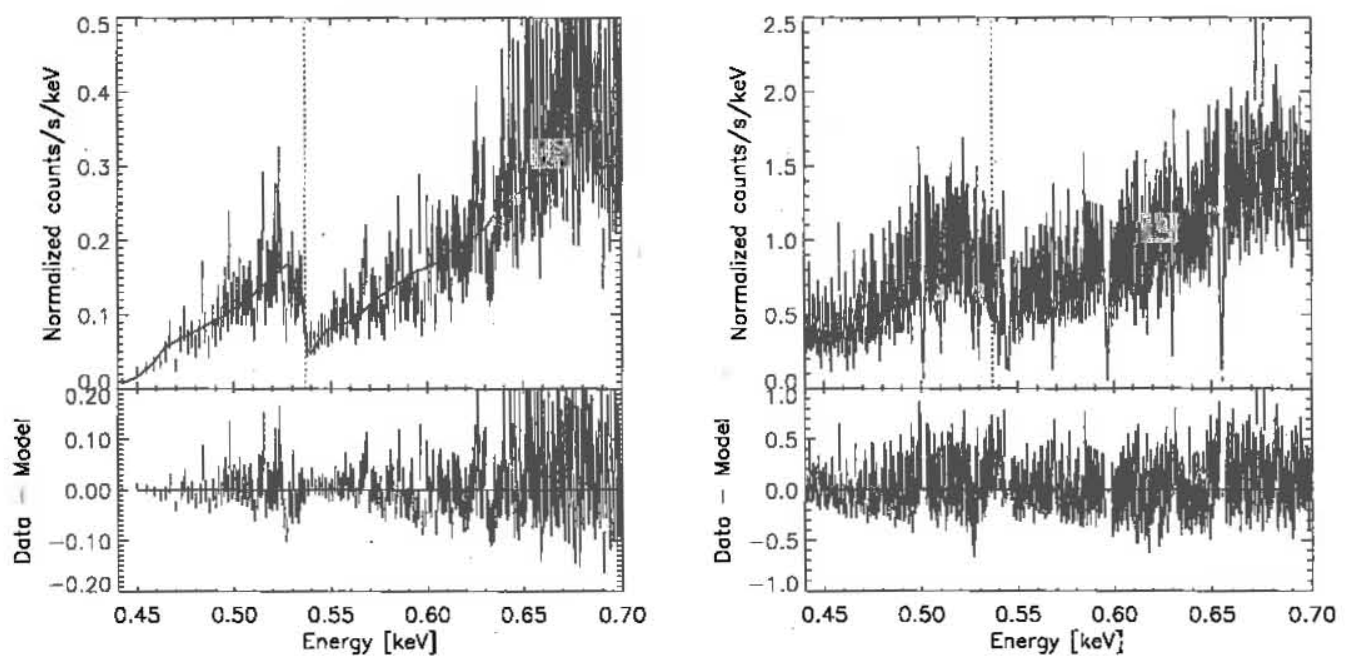

Fig. 1.- Best fits for $\mathrm{O}$ for LETG (left) and RGS (right). The location of the edge is indicated by the dotted line.
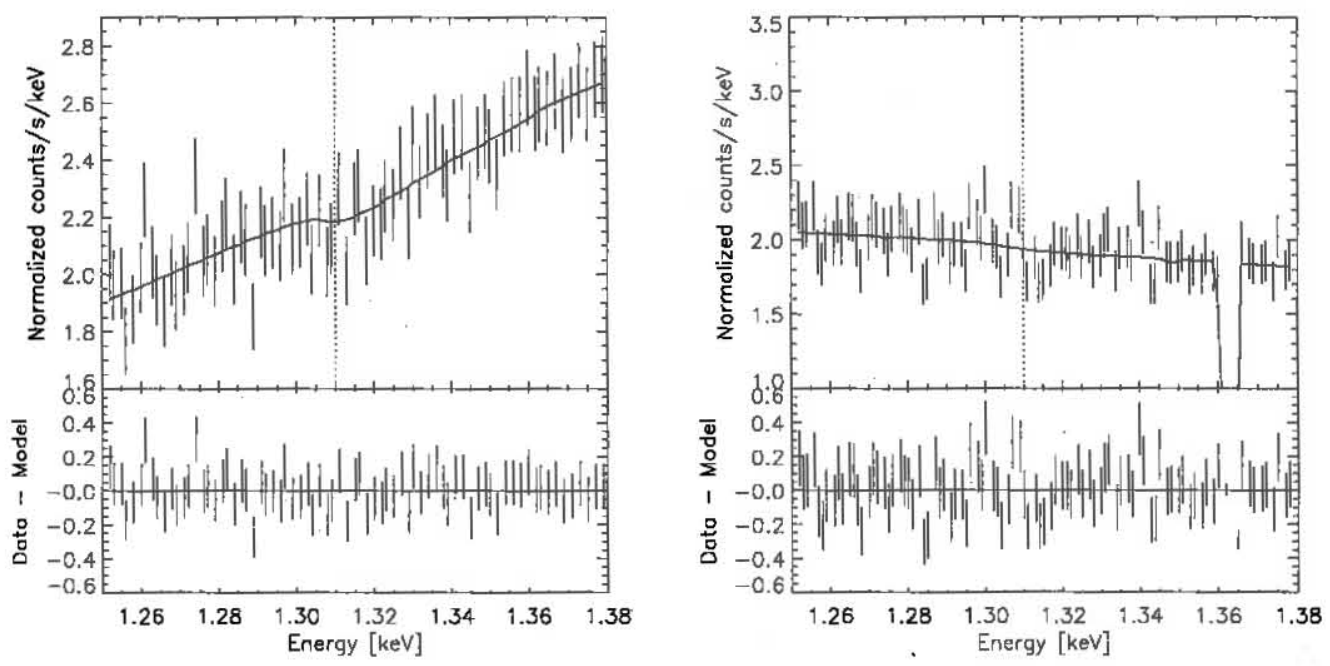

Fig. 2.- Best fits for Mg for LETG (left) and RGS (right). The gap in the RGS spectrum at $1.36 \mathrm{keV}$ is from a bad pixel. The location of the edge is indicated by the dotted line. 


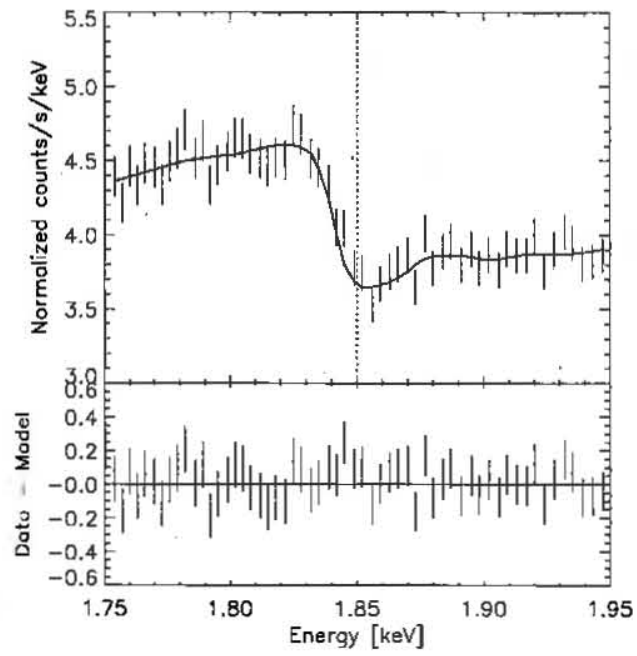

Fig. 3.- Best fit for Si for LETG. The location of the edge is indicated by the dotted line. 

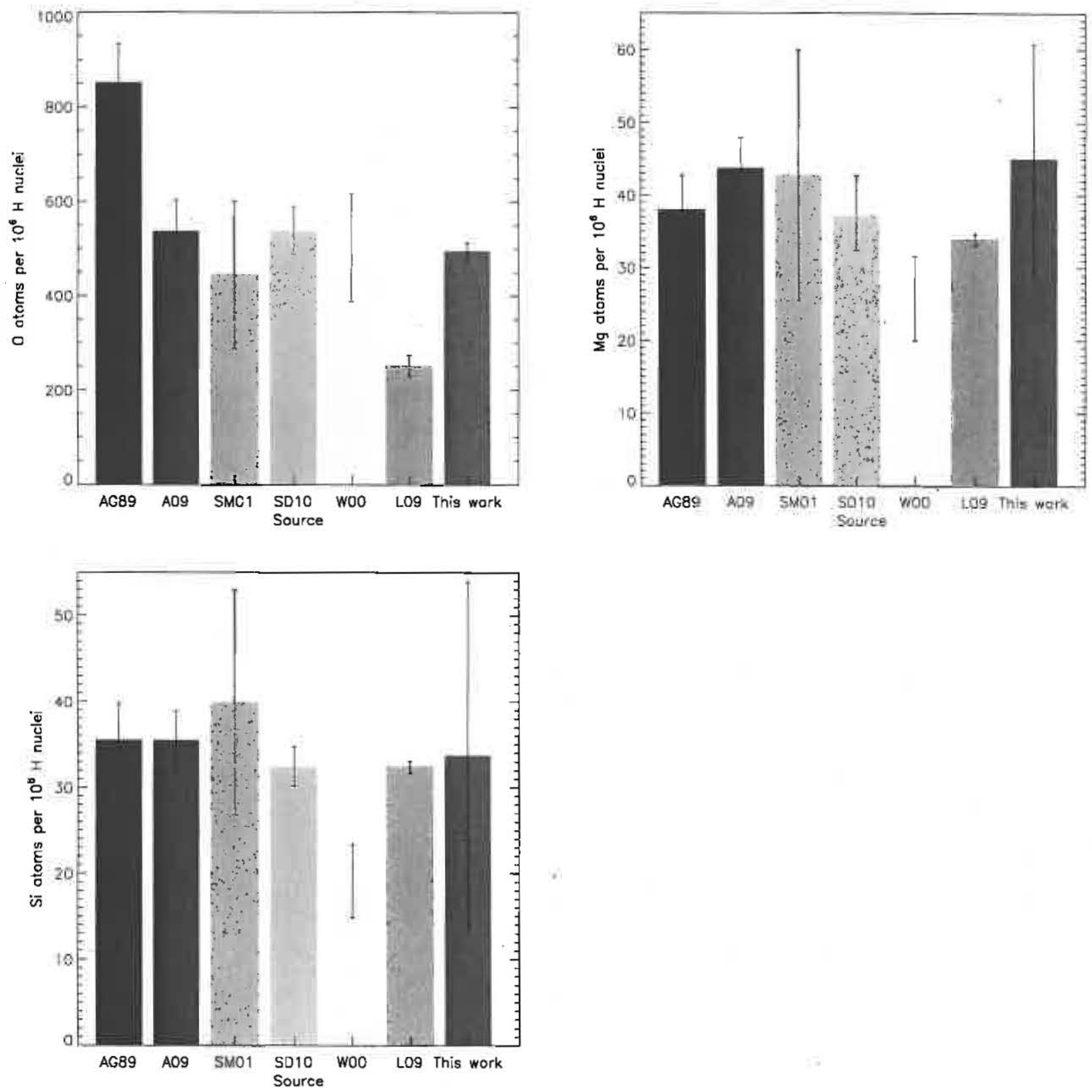

Fig. 4.- Number of $\mathrm{O}, \mathrm{Mg}$, and $\mathrm{Si}$ atoms per $10^{6} \mathrm{H}$ nuclei. Results from other studies are shown for comparison. AG89=Anders \& Grevesse 1989; A09= Asplund et al. 2009; SM01=Sofia \& Meyer 2001; SD10=Simón-Díaz 2010; W00=Wilms et al. 2000; L09=Lodders et al. 2009. 

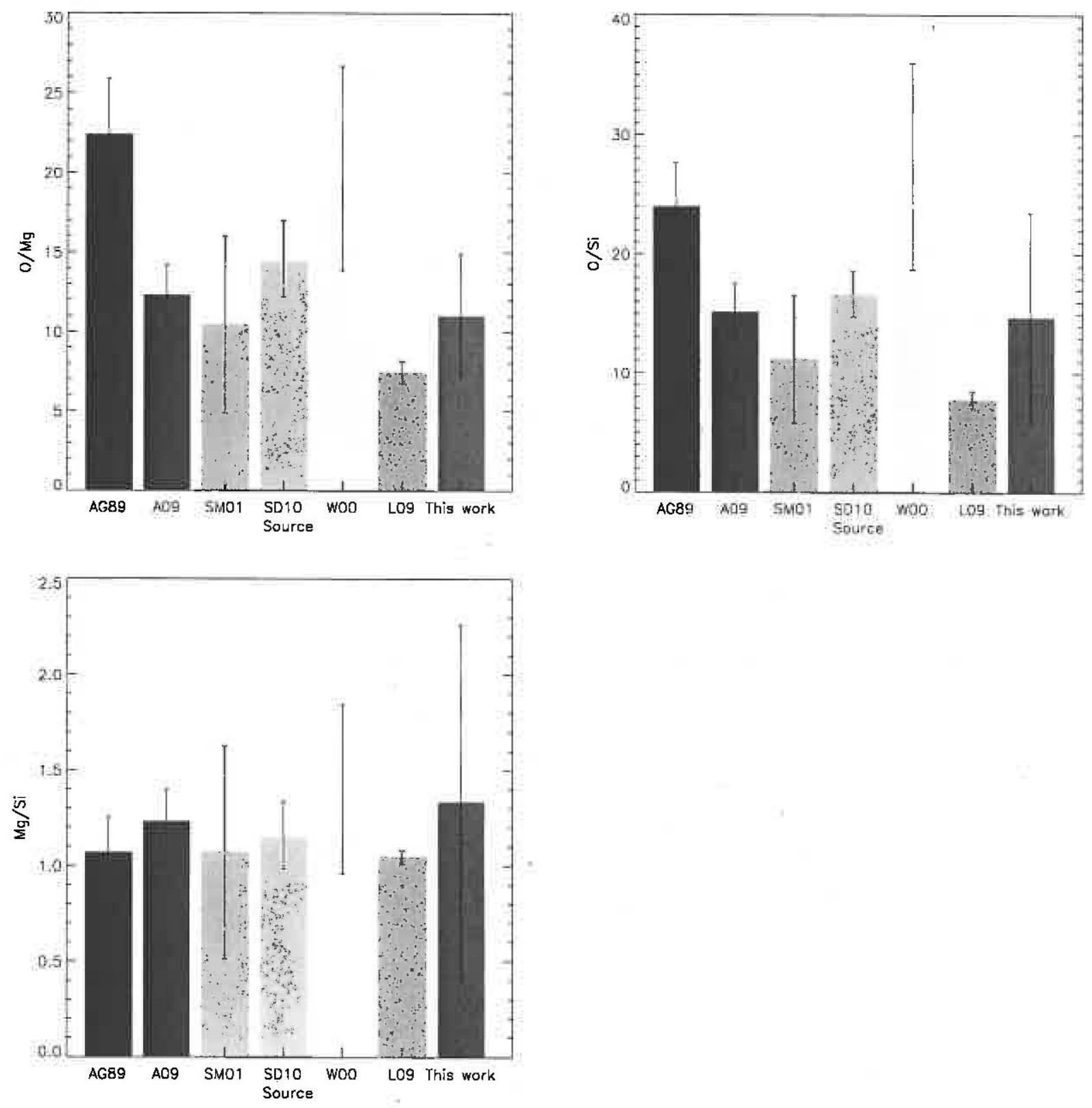

Fig. 5.-- Ratio of $\mathrm{O}, \mathrm{Mg}$, and $\mathrm{Si}$ abundances. Results from other studies are shown for comparison. AG89=Anders \& Grevesse 1989; A09= Asplund et al. 2009; SM01=Sofia \& Meyer 2001; SD10=Simón-Díaz 2010; W00=Wilms et al. 2000; L09=Lodders et al. 2009. 
Table 1: Gas and Dust Phase Abundances of $\mathrm{O}, \mathrm{Mg}$, and $\mathrm{Si}$ Relative to $10^{6} \mathrm{H}$ Nuclei

\begin{tabular}{cccc}
\hline & Total & Gas phase $^{a}$ & Dust phase \\
\hline $\mathrm{O}$ & $495.4 \pm 18.3$ & $308.7_{-20.6}^{+29.8}$ & $186.7_{-27.6}^{+34.5}$ \\
$\mathrm{Mg}$ & $45.0 \pm 15.8$ & $1.24 \pm 0.09$ & $43.8 \pm 15.8$ \\
$\mathrm{Si}$ & $33.65 \pm 20.19$ & $0.92 \pm 0.55$ & $32.73 \pm 20.19$ \\
\hline
\end{tabular}

${ }^{a} \mathrm{O}$ : Jensen et al. 2005; $\mathrm{Mg}$ and Si: Gnacinsiki \& Krogulec 2006 
Table 2: Comparing Grain Model Requirements per $10^{6} \mathrm{H}$ Nuclei and the Dust Phase Abundances

\begin{tabular}{lccc}
\hline \hline Reference & $\mathrm{O}$ & $\mathrm{Mg}$ & $\mathrm{Si}$ \\
\hline Snow \& Witt 1996 & $124-144$ & $20-36$ & $27-40$ \\
Weingartner \& Draine 2001 & $182-193$ & $45-48$ & $45-48$ \\
Zubko et al. 2004 & $114-176$ & $28-34$ & $28-34$ \\
Vaidya et al. 2007 & $(96-112)$ & $(24-28)$ & $24-28$ \\
Iati et al. 2008 & $(180)$ & $(45)$ & 45 \\
Cecchi-Pestellini et al. 2010 & $(136)$ & $(34)$ & 34 \\
This work & 187 & 44 & 33 \\
\hline
\end{tabular}

